\title{
Dynamiques de professionnalisation et jeux de protection : pratiques et enjeux de l'examen des maîtres de chapelle à Rome (1784)
}

The Dynamics of professionalization and the game of protection: the practices and conflicts of the choirmasters in Rome (1784)

\section{Élodie Oriol}

\section{OpenEdition}

\section{Journals}

\section{Édition électronique}

URL : https://journals.openedition.org/ahrf/13418

DOI : $10.4000 /$ ahrf.13418

ISSN : 1952-403X

Éditeur :

Armand Colin, Société des études robespierristes

\section{Édition imprimée}

Date de publication : 15 février 2015

Pagination : 47-69

ISBN : 978-2-200-92958-9

ISSN : 0003-4436

Référence électronique

Élodie Oriol, « Dynamiques de professionnalisation et jeux de protection : pratiques et enjeux de l'examen des maîtres de chapelle à Rome (1784) », Annales historiques de la Révolution française [En ligne], 379 | janvier-mars 2015, mis en ligne le 15 février 2018, consulté le 01 juillet 2021. URL : http:// journals.openedition.org/ahrf/13418; DOI : https://doi.org/10.4000/ahrf.13418 


\title{
DYNAMIQUES DE PROFESSIONNALISATION ET JEUX DE PROTECTION : PRATIQUES ET ENJEUX DE L'EXAMEN DES MAÎTRES DE CHAPELLE À ROME (1784)
}

Élodie ORIOL

\begin{abstract}
À partir de la correspondance de musiciens, cet article propose de reconstruire et d'analyser un conflit né en 1784 autour d'un examen d'entrée pour la fonction de maître de chapelle organisé par la Congregazione dei musici di Roma, institution qui contrôlait la pratique musicale sacrée à Rome. Les étapes du conflit peuvent être reconstruites grâce aux lettres échangées entre Padre Martini, maître de chapelle à Bologne, et deux musiciens exerçant à Rome : Luigi Antonio Sabbatini, l'un de ses anciens élèves et maître de chapelle de l'église des Santi Dodici Apostoli à Rome, et Giovanni Battista Casali, l'un des examinateurs mis en cause. Sabbatini contestait la décision de la congrégation, à savoir le refus d'admettre deux de ses élèves, et dénonçait notamment le rôle joué par Giovanni Battista Casali, qui jouissait alors d'une forte autorité dans le milieu musical romain. Cette affaire éclaire, dans toutes leurs implications, les premiers signes d'une professionnalisation progressive du milieu musical romain au cours de la seconde moitié du XVIII ${ }^{e}$ siècle. En effet, l'affermissement des exigences et du contrôle des qualités musicales passait notamment par la mise en place et l'organisation d'examens. L'étude du conflit, à travers les témoignages des maîtres de chapelle, permet d'entrer au cœur des procédures de qualification et de mettre ainsi au jour les tensions qui pouvaient exister au sein du milieu musical local pour l'accès aux charges les plus rémunératrices, sur le plan matériel comme symbolique. L'examen est révélateur des conflits d'autorité qui traversent ce milieu, des recompositions qu'il favorise et des stratégies mises en œuvre par les musiciens pour asseoir leur position sur la scène urbaine.
\end{abstract}


Il ouvre des pistes de réflexion sur la définition et le partage des codes professionnels, comme sur l'adoption de comportements professionnels collectifs, générés ou modifiés par les formes de qualifications internes introduites au sein des institutions musicales.

Mots-clés : Rome, maîtres de chapelle, conflit, professionnalisation, carrière musicale, expertise.

Dans une lettre datée du 9 juin 1784, le maître de chapelle de l'église des Santi Dodici Apostoli de Rome, Luigi Antonio Sabbatini ${ }^{1}$, informe son homologue bolonais et ancien professeur Giovanni Battista Martini que deux de ses élèves, Giovanni Antonio Ambrosini et Giovanni Francesco Schito, ont soutenu le mois précédent l'examen des maîtres de chapelle de la Congregazione dei musici di Santa Cecilia. Cet examen s'était déroulé le 14 mai 1784 dans l'église de San Carlo ai Catinari, siège de la congrégation, en présence de trois examinateurs : Giovanni Battista Casali, Raimondo Lorenzini et Santi Pesci ${ }^{2}$. Dans sa lettre, Luigi Antonio Sabbatini indique que l'un des examinateurs, Giovanni Battista Casali, a refusé d'admettre les deux candidats, estimant qu'ils ont échoué à l'une des épreuves ; la réponse de la fugue ${ }^{3}$ proposée par les deux candidats n'aurait pas été écrite dans la bonne tonalité. Luigi Antonio Sabbatini conteste ce jugement et, pour convaincre le Cardinal Giovanni Francesco Albani et son neveu Giuseppe Albani, respectivement protecteur et préfet de la congrégation, demande à Giovanni Battista Martini d'examiner les compositions de ses élèves et d'émettre un avis sur leurs qualités :

«Que vous me pardonniez si je vous dérange par la présente, mais je suis très soucieux pour ma réputation $[. .$.$] Voilà pourquoi, je désire [. .$. si j'ai raison, que vous me donniez une réponse claire pour la montrer au Cardinal et à son neveu, et si j'ai tort, je vous prie de la poster ou de

(1) Luigi Antonio Sabbatini (1732-1809) est un compositeur et théoricien de la musique. Il compte parmi les disciples de Giovanni Battista Martini. Depuis 1772, il exerce à Rome en tant que maître de chapelle de l'église des Santi Dodici Apostoli. Il est notamment l'auteur de deux ouvrages théoriques : Elementi Teorici della Musica, Rome, 1789-1790 et Trattato Sopra le Fughe Musicali,Venise, 1802. Voir la notice de Sven Hansell dans le Grove Music Online.

(2) La date précise de l'examen est indiquée dans la lettre de Luigi Antonio Sabbatini datée du 14 juillet 1784 adressée à Giovanni Battista Martini conservée au Museo internazionale e Biblioteca della musica di Bologna, Carteggio di Padre G. B. Martini. Par la suite, le fonds de conservation des lettres provenant de la correspondance de Padre Martini ne sera plus précisé.

(3) En musique, la fugue commence par l'exposition d'un thème, dit « sujet », suivi de la « réponse », qui est le sujet répété au ton, soit de la dominante, soit plus rarement de la sous-dominante. 
la retourner de quelque façon que ce soit [...] pour soutenir ma dignité, tandis que le dit Casali, sur toutes les places de Rome, propage partout que l'affaire va assez mal, et cette rumeur se répand à Rome, aussi bien dans la profession qu'à l'extérieur, qu'on ne parle de rien d'autre que de ce sujet $»^{4}$.

Dès le début de la lettre, on saisit l'enjeu de l'initiative de Sabbatini : rétablir, par le recours à une autorité externe, sa réputation localement compromise. Alors qu'on débat ardemment de cette affaire à Rome aussi bien dans le milieu professionnel que sur la place publique, Sabbatini s'adresse à Padre Martini pour qu'il fasse une contre-expertise, confiant dans ses compétences reconnues et supérieures. Ce soutien permettrait de défendre l'honneur du professeur des deux candidats recalés. Dans sa réponse datée du 16 juin 1784, Martini informe Sabbatini qu'il accepte de se charger de l'affaire et annonce qu'il écrira sans tarder à Casali à ce sujet $^{5}$. La mort de Martini, au début du mois d'août, complique cependant les initiatives de Sabbatini. Ce dernier écrit néanmoins dans les mois qui suivent à Stanislao Mattei, un proche de Martini, pour poursuivre cette affaire qui, alors, n'a toujours pas été résolue.

Commencés ainsi en juin 1784, les échanges épistolaires des trois maîtres de chapelle dureront un peu plus de deux mois. Les documents que l'on se propose d'étudier font partie de la collection des correspondances de Giovanni Battista Martini conservée au Museo internazionale e Biblioteca della musica di Bologna. Si plusieurs spécialistes, conscients de l'intérêt de cette source pour l'histoire de la musique du XVIII ${ }^{\mathrm{e}}$ siècle, l'ont examinée selon des perspectives de recherche variées (étude de la pratique musicale, constructions biographiques, réseaux de musiciens... $)^{6}$, son exploitation reste encore partielle. Certaines lettres tirées de la correspondance entre Martini et Sabbatini, qui n'a fait l'objet d' aucune étude systématique, seront ici étudiées dans la mesure où elles éclairent les transformations à l'œuvre dans le milieu musical romain.

(4) Lettre de Luigi Antonio Sabbatini à Padre Martini, Rome, 9 juin 1784.

(5) Lettre de Giovanni Battista Martini à Luigi Antonio Sabbatini, Bologne, 16 juin 1784.

(6) Sur la correspondance de Giovanni Battista Martini, voir Anne ScHNOEBELEN, Padre Martini's Collection of Letters in the Civico museo bibliografico musicale in Bologna: an annotated index, New York, Pendragon Press, 1979 ; Giancarlo RostiROLLA, « La corrispondenza fra Martini e Girolamo Chiti : una fonte precioza per la conoscenza del Settecento musicale italiano », dans Angelo PAMPILIO (dir.), Padre Martini. Musica e cultura nel Settecento europeo, Florence, Olschki, 1987, p. 236-276. Les lettres échangées entre Girolamo Chiti et Giovanni Battista Martini ont récemment été publiées par Giancarlo RoSTIROLLA, Luciano LUCIANI, Maria Adelaide MORABITO IANNUCCI et Cecilia PARISI (dir.), Epistolario Giovanni Battista Martini e Girolamo Chiti (1745-1759). 472 lettere del museo internazionale e biblioteca della musica di Bologna, Rome, Ibimus, 2010. 
Cet article n'a pas pour objectif de revenir sur les points techniques sur lesquels débattent les trois maîtres de chapelle, mais d'observer en quoi ce conflit, né autour d'une procédure d'examen, révèle les tensions qui traversent le milieu musical au sujet des procédures de qualification et de l'accès aux charges les plus rémunératrices, tant sur le plan matériel que symbolique.

En effet, l'exercice de la musique dans les lieux de culte est régulé par la Congregazione dei Musici di Santa Cecilia di Roma qui, depuis le début du XVIII ${ }^{\mathrm{e}}$ siècle, dispose d'un monopole réglé par des statuts reconnus par les autorités pontificales. Le conflit qui s'ouvre en $1784 \mathrm{au}$ sujet d'un examen organisé par cette institution permet de voir comment l'introduction ou l'amélioration des contrôles de compétences musicales, signes d'une professionnalisation progressive ${ }^{7}$, modifient l'organisation du milieu musical, produisent des discours révélateurs des représentations associées à la musique et à ses métiers, et offrent de nouveaux espaces aux stratégies d'affirmation professionnelle ${ }^{8}$. L'épisode conflictuel s'inscrit en effet dans le processus de professionnalisation des structures et des pratiques musicales qui marque le $\mathrm{XVIII}^{\mathrm{e}}$ siècle, en particulier dans sa seconde moitié ${ }^{9}$, et qui a été peu étudié pour la ville de Rome ${ }^{10}$. La pratique de l'examen qui, à première vue, peut se présenter comme un mode de sélection juste, car reposant sur des démonstrations objectives de compétences, vérifiées par la communauté professionnelle dont elles renforcent la cohésion, modifie en réalité le jeu des protections et des recommandations sans les neutraliser. Choisir comme angle d'approche les pratiques d'examen, élément significatif d'une professionnalisation en cours, permet de saisir les évolutions qui conduisent à modifier le statut

(7) Sur les marqueurs de la professionnalisation observés dans les milieux artistiques, voir notamment Bram KEMPERS, Peinture, pouvoir et mécénat. L'essor de l'artiste professionnel dans l'Italie de la Renaissance, Paris, Monfort, 1997 ; Nathalie HEINICH, Du peintre à l'artiste, artisans et académiciens à l'âge classique, Paris, Éditions de Minuit, 1999.

(8) À titre comparatif, voir l'étude conduite sur les stratégies des écrivains professionnels, par Alain Viala, La naissance de l'écrivain, Paris, Éditions de Minuit, 1985, p. 170-289.

(9) Pour des éclairages sur la professionnalisation de la vie musicale en Europe, voir notamment Hans Erich BöDEKER, Patrice VEIT et Michael WERNER (dir.), Les sociétés de musique en Europe, 1700-1920 : structures, pratiques musicales, sociabilités, Berlin, Berliner-Wissenschafts-Verlag, 2007. Pour une étude de ce processus dans la ville de Paris, voir David HenNEBELLE, De Lully à Mozart. Aristocratie, musique et musiciens à Paris (XVII ${ }^{e}$ XVIII ${ }^{e}$ siècles), Seyssel, Champ Vallon, 2009.

(10) Ce dossier est issu de ma thèse de doctorat en histoire moderne intitulée «Vivre de la musique à Rome au XVIII ${ }^{\mathrm{e}}$ siècle : lieux, institutions et parcours individuels », Aix-Marseille Université - Università di Roma I, « La Sapienza », sous la direction de Renata Ago et Brigitte Marin, soutenue le 6 décembre 2014 à Aix-Marseille Université. 
du musicien professionnel dans la société de l'époque, dont l'impact est particulièrement fort dans la seconde partie du siècle.

\section{La pratique de l'examen des maîtres de chapelle : histoire, signification et enjeux}

\section{L'examen, un sésame pour entrer dans la congrégation des musiciens romains}

Depuis 1716, pour pouvoir exercer dans les édifices sacrés, les musiciens doivent obligatoirement être membres de la Congregazione dei Musici di Santa Cecilia di Roma ${ }^{11}$. Cette confrérie, animée de finalités corporatives, professionnelles et religieuses, règle l'exercice de la musique sacrée à Rome. Il s'agit d'une institution laïque dont les membres sont ecclésiastiques ou non, et mixte, bien qu'il faille attendre 1774 pour qu'une femme, la compositrice Maria Rosa Coccia, y soit admise. Seule la chapelle pontificale échappe à son contrôle et fonctionne comme une institution indépendante.

La confrérie réunit ainsi les musiciens qui pratiquent la musique régulièrement ou occasionnellement dans les institutions musicales religieuses de Rome, à savoir les grandes ou petites chapelles des basiliques, les églises, les collèges, les couvents, les pensionnats, les orphelinats, les oratoires, les confréries, les écoles religieuses... Cette institution répartit ses membres en quatre catégories : les maîtres de chapelle (maestri di cappella), les organistes (organisti), les instrumentistes (strumentisti) et les chanteurs (musici). À la tête de chacune d'elles se trouve un gardien (guardiano) dont la charge annuellement renouvelée est centrale dans le cadre institutionnel de la confrérie.

Les derniers statuts de l'institution validés par le Pape Innocent XI en 1716 ne se prononcent que timidement sur l'organisation des examens d'entrée. Les chapitres XXI et XXII indiquent sommairement que le musicien qui souhaite exercer dans les chapelles musicales doit être obligatoirement 《abilitato e approvato per idoneo $»^{12}$ par quatre maîtres

(11) La Confraternita musicorum de Urbe naît officiellement le $1^{\mathrm{er}}$ mai 1585 par décret apostolique. En promulguant la bulle Rationi congruit, le pape Sixte Quint reconnât l'existence d'un noyau déjà actif de musiciens opérant à Rome, connu sous le nom de Compagnia dei Musici di Roma. La confrérie musicale nouvellement créée est placée sous la protection de la Vierge Marie, saint Grégoire le Grand et sainte Cécile. Sur l'histoire de la confrérie voir notamment l'ouvrage de Remo GiAZotTo, Quattro secoli di Storia dell'Accademia Nazionale di Santa Cecilia, Rome, Accademia Santa Cecilia, 1970, 2 vol.

(12) Archivio dell' Accademia di Santa Cecilia, Carteggio, Serie II. 
de chapelle élus tous les ans qui prennent la fonction d'examinateur. Rien n'est cependant précisé sur le contenu et le déroulement des examens.

L'étude des comptes rendus d'assemblée de la confrérie permet d'observer que ses membres portent progressivement une plus grande attention à l'amélioration du fonctionnement de l'examen au sein de l'institution. Ces initiatives vont dans le sens d'une professionnalisation accrue de la pratique musicale, par le contrôle de la formation et de la qualité du travail.

En juillet 1741, devant le constat qu'un certain nombre de maîtres de chapelle exercent sans avoir passé l'examen, on décide que les maîtres non habilités obtiendraient la patente à titre gracieux, mais, qu'à l'avenir, tous les futurs candidats devront se soumettre à l'examen de contrepoint et de chant ecclésiastique ${ }^{13}$.

Signes de l'attention plus vive qu'ils portent à l'examen d'entrée, les membres de la confrérie se soucient de la fonction d'examinateur. Ils prennent des mesures pour la rendre plus efficace et pour faciliter la procédure de désignation. C'est en effet sur les examinateurs que repose la responsabilité des nouvelles admissions des maitres de chapelle qui souhaitent exercer dans les différentes institutions religieuses. Ces examinateurs, à ce moment fixé au nombre de cinq (quatre maîtres de chapelle et le gardien en fonction), doivent occuper leur charge pendant l'année entière. En cas d'absence, l'assemblée doit élire un remplaçant ${ }^{14}$.

En 1747, Girolamo Chiti, maître de chapelle exerçant à Rome, explique à Martini le fonctionnement de l'examen pour devenir maître de chapelle :

« Je sais de façon certaine par Pitoni, et selon une ancienne et inaltérable habitude, que pour l'examen des maitres de chapelle de toutes sortes, et pour les concours qui se font à Rome, cela fonctionne et fonctionnera toujours avec la proposition d'une antienne en cantus firmus, pour voir si le candidat connaît son devoir ; règle qui nous a été donnée par Palestrina et par les observations d'Antimo Liberati etc. etc. etc $»^{15}$.

(13) Ibidem, Atti delle congregazioni, Registro 4, 18 juillet 1741.

(14) Ibid., Registro 4, 21 avril 1746.

(15) Lettre de Girolamo Chiti à Giovanni Battista Martini, Rome, 6 septembre 1747, dans Giancarlo Rostirolla, Luciano LUCIANI, Maria Adelaide MORABITO IANNUCCI et Cecilia PARISI (dir.), op. cit., p. 295. 
Pour leurs examens d'entrée, les compositeurs doivent donc composer une antienne ${ }^{16}$ en cantus firmus. Chiti défend cette procédure et rappelle que c'est une règle de composition donnée par Palestrina et confirmée par les observations d'Antimo Liberati (1617-1792) $)^{17}$ qui, au XVIII' ${ }^{\mathrm{e}}$ siècle, jouit d'un grand crédit chez les défenseurs de la «Scuola Romana $»^{18}$. Selon Arnaldo Morelli, l' « école musicale romaine » reposait, depuis la fin du XVII ${ }^{\mathrm{e}}$ siècle, sur le modèle de la pratique musicale de la chapelle pontificale et de ses compositeurs, en premier lieu Palestrina, autour duquel se construisit un véritable mythe.

Malgré les initiatives répétées des membres de la confrérie pour faire respecter l'admission obligatoire et le passage de l'examen, les différents rappels qui apparaissent dans les comptes rendus d'assemblée révèlent que, pendant tout le siècle, des musiciens continuent d'exercer sans avoir la patente et sans être inscrits au sein de la confrérie ${ }^{19}$.

En mai 1784, les deux élèves de Luigi Antonio Sabbatini sont pourtant bien convoqués pour passer un examen d'admission. L'étude des lettres échangées entre les trois compositeurs au sujet de leur éviction met en lumière les enjeux que pouvait recouvrir la pratique de l'examen.

\section{L'examen ou l'expression de l'autorité des musiciens}

Pour les musiciens, la pratique de l'examen devient un moyen d'affirmer leur autorité dans le milieu professionnel, et ceci dans les différentes fonctions qu'ils occupent. Suivant cette logique, l'échec d'un élève dans ce cadre peut ternir la réputation de l'enseignant. Sabbatini, en tant que professeur des deux candidats, explique clairement que l'exclusion de ses élèves conduit à le discréditer. Ce refus d'admission porte atteinte à sa personne dans l'exercice de sa fonction d'enseignant. Il présente

(16) Selon Rousseau, la définition de l'antienne est la suivante : « Sorte de Chant usité dans l'Église Catholique. [...] Aujourd'hui, la signification de ce terme est restrainte à certains passages courts de l'Ecriture, qui convienne à la Fête qu'on célèbre, \& qui précédant les Pseaumes \& Cantiques, en réglant l'intonation »(Jean-Jacques RouSSEAU, Dictionnaire de la musique, Paris, Chez la veuve Duchesne, 1768, p. 32).

(17) Les écrits théoriques d'Antimo Liberati (1617-1692), chanteur et compositeur né à Foligno, défendaient notamment la place de Palestrina et le qualifiaient de « sauveur » de la polyphonie.

(18) Sur l'acception de cette expression, voir notamment Arnaldo MoRELLI, "Schola romana", "Stile di cappella" e cerimoniale papale », dans Musici e istituzioni musicali a Roma nello stato pontificio nel tardo Rinascimento : attorno a Giovanni Maria Nanino, Atti della Giornata di studi (Tivoli, Villa d'Este, 26 ottobre 2007), vol. 81, $\mathrm{n}^{\circ} 1,2008$.

(19) Sur le travail en dehors du cadre corporatif, voir Steven KAPLAN, « Les corporations, les "faux ouvriers" et le faubourg Saint-Antoine au XVIII" siècle », Annales ESC, 1988, nº 2, p. 353-378; Michel Sonenscher, Work and Wages. Natural Law, Politics and the Eighteenth Century French Trades, Cambridge, Cambridge University Press, 1992. 
ainsi son initiative comme étant un moyen de défendre sa « réputation », de retrouver « honneur » et « dignité ». On rencontre explicitement cette revendication dans une lettre adressée à Stanislao Mattei en décembre 1784. Sabbatini indique clairement pourquoi, malgré le décès de Padre Martini, il tient à poursuivre son engagement dans cette affaire :

« Très cher Père Mattei, cette affaire me tient très à cœur, pas seulement pour les jeunes gens, mais pour ma réputation, et celle de notre école, et ces messieurs les Académiciens devraient être de cet avis $»^{20}$.

Le musicien précise qu'il défend ses élèves non seulement pour protéger sa propre réputation, mais aussi celle de l'école bolonaise à laquelle il est attaché comme ancien élève de Martini, l'un de ses principaux représentants.

La mise en place de l'examen peut également devenir un moyen de favoriser un certain conservatisme, ou du moins une fermeture corporative, en protégeant certains musiciens au détriment d'autres qui se trouvent ainsi écartés et empêchés de développer leurs activités. Cette utilisation est visible aussi bien à Rome qu'à Bologne.

Dans la première lettre qu'il envoie à Casali, Padre Martini indique que le conflit qui concerne les élèves de Sabbatini lui fait penser à la procédure d'admission de ce dernier à l'Accademia Filarmonica di Bologna. Celle-ci ne s'est visiblement pas faite sans difficulté et Martini rappelle ainsi au compositeur romain la confiance qu'il lui a accordée dans le passé, sous-entendant par la même occasion qu'il lui en est débiteur :

«En écoutant cette controverse, je me suis souvenu de ce qu'il advint en 1740 de votre illustrissime, lorsque vous vous présentâtes à l'examen pour devenir membre de l'Accademia Filarmonica di Bologna ; sur le fait qu'ils ne voulurent pas vous admettre, disant que la composition était mal faite et pleine d'erreurs [...] Mais comment ! Vous vous souviendrez sans doute très bien, que la raison n'était pas que la composition fut mal faite, mais c'était votre personne qu'ils craignaient voir s'installer à Bologne, leur enlevant des fonctions musicales, et c'est pourquoi ils étaient conduits non par la raison, mais par la passion $»^{21}$.

Martini témoigne ainsi sans ambiguïté des manœuvres des maîtres de chapelle en fonction à Bologne, qui avaient alors utilisé l'examen

(20) Lettre de Luigi Antonio Sabbatini à Stanislao Mattei, Rome, 25 décembre 1784.

(21) Lettre de Giovanni Battista Martini à Giovanni Battista Casali, Bologne, sans date. 
pour limiter la concurrence dans la ville. Ils souhaitaient empêcher Casali d'exercer par crainte de voir diminuer le nombre de leurs engagements et perdre ainsi une partie de leurs prérogatives.

À Rome, la pratique de l'examen semble également avoir été un moyen, pour Casali, de conforter une position d'autorité. Ce dernier devient au sein de la Congregazione dei musici di Santa Cecilia di Roma un élément structurant et une référence dans le milieu musical local. Il occupe en effet à plusieurs reprises la charge de gardien de la catégorie des maestri di cappella $(1752-1753 ; 1760-1761 ; 1771-1772 ; 1779-1791)^{22}$ et exerce dans un grand nombre de chapelles musicales ${ }^{23}$. Selon Sabbatini, si Casali occupe une position dominante à Rome, c'est en particulier grâce à la fonction d'examinateur qu'il occupe. L'exercice de cette charge lui confère en effet une qualité de juge en matière musicale et lui permet de contrôler les nouvelles admissions dans la ville.

Dans une lettre datée du 3 juillet 1784, Sabbatini indique que la situation s'envenime, notamment à cause de l'influence prédominante de Casali dans le milieu musical romain :

« La Profession, ici à Rome, est gérée par un coquin, et pour toutes les affaires il fait montre de deux visages, et veut que les choses se fassent à sa manière, avec grande perfidie [...] Ici, à Rome, ils le prennent pour un oracle, et celui-ci s'en est rendu compte, et c'est pour cela qu'il fait le coq ; cela serait l'occasion de le mortifier et d'enlever un peu l'arrogance qu'il a dans la tête $[\ldots]{ }^{24}$.

Le 10 juillet 1784, Sabbatini se plaint de la toute puissance de Casali et reporte l'opinion d'un anonyme qui ternit la réputation de Martini en retour :

« Lequel [Casali] s'est constitué tout seul ici à Rome un royaume sur la musique, et tous dépendent de lui, de sorte qu'il y a quelqu'un qui, dans cette circonstance, s'est exprimé ainsi : que veut dire Martini ? Pourquoi

(22) Voir la notice "CASALI Giovanni Battista", Dizionario Biografico degli Italianion lineVolume 21 (1978), notice de Maria Caraci.

(23) Giovanni Battista Casali cumule déjà un grand nombre de postes dans les chapelles musicales en 1754, alors qu'il se présente à un poste de maître de chapelle dans l'église de la Chiesa Nuova, comme l'atteste la liste qu'il dresse des églises dans lesquelles il exerce. En plus d'être le maître de chapelle suppléant dans la basilique San Giovanni in Laterano et à San Lorenzo in Damaso, le musicien exerce dans vingt-deux autres églises. Document cité par Bianca Maria ANTOLINI, «La vita musicale a Roma nella seconda metà del Settecento. Nuove acquisizioni e prospettive di ricerca », Analecta musicologica, ${ }^{\circ} 45,2011$, p. 328-360, p. 329.

(24) Lettre de Luigi Antonio Sabbatini à Giovanni Battista Martini, Rome, 3 juillet 1784. 
veut-il porter un jugement contre les raisons de Casali ? Alors que quand Martini lui-même dirigea sa musique ici à Rome, il alla d'abord voir Casali. Voyez maintenant quelle estime on vous porte, et quel monarque est celuici, mais ils n'omettent pas d'ailleurs de le traiter de coquin dans certaines occasions $»^{25}$.

Pour Sabbatini, Casali est le principal responsable du climat délétère qui règne à Rome, plus spécifiquement au sein de la Congrégation de Sainte Cécile, dans laquelle il a les moyens de contrôler les admissions, et à travers elles d'amples segments de l'accès au travail des musiciens sur le marché urbain.

Dans les archives de la chapelle de San Giovanni in Laterano se trouve un document anonyme qui confirme la position dominante de Casali et dénonce la cooptation comme mécanisme de recrutement au sein de la Congrégation. Ce document intitulé Memoriale per il Maestro Giuseppe Heiberger a été rédigé en 1775, au moment où Giuseppe Heiberger ${ }^{26}$ se présente au poste de coadiutore de Giovanni Battista Casali, maître de chapelle titulaire de San Giovanni in Laterano ${ }^{27}$. L'auteur anonyme indique que Casali a refusé de reconnaître les compétences de Heiberger au seul motif qu'il a préféré demander l'admission à la Filarmonica di Bologna plutôt que de passer l'examen de la Congregazione dei Musici di Santa Cecilia. Certains arguments avancés par l'auteur anonyme portent sur les mécanismes de sélection mis en oeuvre. Des maestri se réservent exclusivement le choix des musiciens à promouvoir, sans autre consultation externe, et de la sorte, privatisent l'institution. Pour l'auteur de ce mémorial « l'examen a toujours été arbitraire et partial », les examinateurs se montrant bien plus tolérants avec les candidats qu'ils soutiennent. La procédure de l'examen vient en quelque sorte masquer les véritables ressorts des recrutements, à savoir les liens tissés entre les maestri de l'institution et leurs protégés ; il s'agit d'une simple cooptation ${ }^{28}$.

(25) Lettre de Luigi Antonio Sabbatini à Giovanni Battista Martini, Rome, 10 juillet 1784. Le passage a été souligné par l'auteur.

(26) La carrière de ce compositeur est peu connue. Son nom figure néanmoins dans les journaux et les périodiques de l'époque. L'un de ses opéras a fait par exemple l'objet d'une critique positive dans la Gazzetta Universale du 18 février 1777. Voir Franco PIPERNO, « Le système de production jusqu'en 1780 », dans Lorenzo BIANCONI et Giorgio PESTELLI (dir.), Histoire de l'opéra italien. Le système de production et ses implications professionnelles, Liège, Mardaga, 1992, vol. 4, p. 71.

(27) ASVR, Capitolo di San Giovanni in Laterano, Urbis = Cappella Pia Lateran (1766-1866), Urbis 13, O LXXXIX, ${ }^{\circ}$ 3, Urbis 1775.

(28) Sur ce mode de recrutement, voir Mark GRANOVETTER, Getting a Job: A Study of Contacts and Careers, Chicago, University of Chicago Press, 1995. 
La rigidité dont fait preuve la Congregazione dei Musici di Santa Cecilia pour les examens d'entrée des compositeurs peut ainsi être interprétée autrement que par la seule volonté de relever le niveau de qualification des musiciens et par une nouvelle attention aux exigences de la profession. Elle devient aussi un moyen de limiter la concurrence et, pour certains musiciens comme Casali, d'étendre une influence dans le milieu local en tenant fermement en main l'accès à l'emploi au profit d'élèves fidèles. Un signe de fermeture, donc, plutôt que d'ouverture sur un recrutement élargi et de qualité par l'adoption de normes et d'exigences propres à la profession.

À travers le conflit qui naît de l'examen des deux élèves de Sabbatini, on peut également voir se dessiner les différences de positionnement et d'opinion autour du jugement esthétique dans le domaine musical ${ }^{29}$. Dans la première lettre qu'il adresse à Casali qui associe marques d'autorités, conseils amicaux et menaces à peine voilées, Giovanni Battista Martini donne une définition précise de l'expertise musicale et des valeurs qui, selon lui, doivent lui être attachées :

«J'ai appris qu'on souhaite que je prenne la défense des candidats, et que je fasse voir au public le tort que vous leur avez fait. Très estimé Monsieur Casali, être juge et examinateur est une chose en soi honorifique, et respectable, mais à bien y penser, les qualités sont trop nombreuses pour qui doit vraiment juger, et parce que le juge doit être informé de toutes les opinions qui se trouvent dans son Art, alors que le candidat a le droit de résister à n'importe quelle sentence, à moins qu' elle ne soit universellement interdite ou condamnée, et l'art de la fugue est complexe, et les façons d'y répondre sont nombreuses. Dans cette affaire, vous avez perdu la cause, et vous perdrez encore votre honneur et votre dignité, si bien que je vous conseille de vous retirer de votre engagement de ne pas vouloir accepter les candidats, de prendre le risque de les accepter et sauver ainsi votre dignité.

(29) Pour un ouvrage récent sur les formes d'expertise voir notamment Christelle RABIER (dir.), Fields of Expertise. A Comparative History of Expert Procedures in Paris and London, 1600 to present, Newcastle-upon-Tyne, Cambridge Scholars Publishing, 2007. Sur l'expertise dans le domaine artistique, voir Raymonde MOULIN et Alain QUEMIN, « La certification de la valeur de l'art. Experts et expertises », Annales ESC, nov.-déc. 1993, nº 6, p. 1421-1445 ; Charlotte GuICHARD, « Les formes de l'expertise artistique en Europe (XIV ${ }^{\mathrm{e}}-\mathrm{XVIII}^{\mathrm{e}}$ siècle) », Revue de Synthèse, 2011, vol. 132, ${ }^{\mathrm{o}} 1$, p. 1-11; Mélanie TRAVERSIER, « De l'érudition à l'expertise : Saverio Mattei (1742-1795), "Socrate imaginaire" dans la Naples des Lumières », Revue historique, 2007, vol. 2007/1, n 641, p. 91-136. 
Parce que je vous assure que vous ferez mauvaise figure en étalant cette controverse par la presse dans toute l'Europe $»^{30}$.

Émettre un jugement est ainsi perçu comme un honneur qui contribue à la dignité de la profession. Martini développe ici un argumentaire sur le jugement esthétique qui peut être rapproché de l'idéal véhiculé par la République des Lettres ${ }^{31}$. Parmi les critères retenus par le compositeur bolonais, on trouve la dimension collective du jugement et la conscience de l'universel. Le jugement ne peut être établi par un seul individu. Il est nécessaire que ce dernier s'informe auprès de la communauté de savants qui représente l'art en question. Martini compte ainsi convaincre Casali d'écouter les remarques d'autres experts et indique que les candidats ont le droit de contester une décision qui n'a pas été prise de façon collégiale. Bien que Martini approuve et reconnaisse la valeur du jugement esthétique en matière musicale, il récuse toute forme d'exclusivité ou de monopole exercée dans ce cadre. La réaction de Casali est contraire aux principes d'universalisme et de communauté de talents, égaux dans leurs fonctions et leurs capacités à juger, qu'il défend pour construire une identité collective professionnelle qui dépasse les particularismes et les ancrages locaux.

\section{Les stratégies des musiciens face aux évolutions du milieu musical}

À partir des correspondances, il est possible d'observer les différentes stratégies mises en œuvre par les musiciens engagés dans ce conflit singulier, et d'évaluer ainsi des différences de positionnement révélatrices de conceptions divergentes de la profession, à un moment où le milieu musical romain se recompose, en particulier par le succès de nouveaux genres musicaux. Si Rome se montre relativement ouverte aux musiciens étrangers qui peuvent être admis dans la Congrégation de Sainte Cécile, les postes stables et rétribués dans les chapelles restent comptés et par conséquent enviés ${ }^{32}$. En plus de conférer une situation économique privilégiée, ces postes de maîtres de chapelle donnent l'occasion de se faire connaître,

(30) Lettre de Giovanni Battista Martini à Giovanni Battista Casali, Bologne, non datée (16 juillet 1784 env.).

(31) Voir en particulier Françoise WAQUET, «Qu'est-ce que la République des Lettres ? Essai de sémantique historique », Bibliothèque de l'École des chartes, 1989, vol. 147, p. 473-502.

(32) Sur l'intégration des musiciens étrangers à Rome au XVIII ${ }^{\mathrm{e}}$ siècle, voir notamment Élodie ORIOL, « Musicisti e ballerini stranieri a Roma: percorsi sociali e sviluppo delle carriere nella prima metà del Settecento » dans Anne-Marie GoULET, Gesa ZUR NIEDEN (dir.), Europaische Musiker in Venedig, Rom und Neapel, actes du colloque conclusif de l'ANR-DFG MUSICI, Rome, 19-21 janvier 2012, Analecta musicologica, vol. 52, 2014, p. 269-299. 
d'être sollicités pour d'autres prestations musicales et d'étendre ainsi leur réseau professionnel. Enfin, si le succès de la musique théâtrale génère effectivement d'autres canaux de réussite dans la ville, les perméabilités et les porosités entre musique sacrée et musique profane, les circulations des musiciens entre chapelles et théâtres rendent ces postes attractifs pour se situer en bonne place sur le marché musical romain dans toutes ses composantes.

\section{Luigi Antonio Sabbatini : solliciter la reconnaissance des pairs}

Le professeur des deux étudiants recalés, Luigi Antonio Sabbatini, construit sa défense en ayant recours à des procédés multiples pour mieux faire entendre sa voix. Il mobilise aussi bien des ressources locales que des ressources externes pour contrer le jugement de Casali.

Le recours à une personnalité de référence comme Giovanni Battista Martini se présente comme une recherche de légitimation extérieure pour promouvoir la carrière de ses élèves dans le système local. Le musicien est conscient de l'autorité qu'exerce le compositeur bolonais et espère que son appui l'accréditera auprès de la communauté musicale ${ }^{33}$. Auteur d'un Saggio fondamentale pratico di contrapunto sopra il canto fermo (Bologne 1774, 2 tomes) et de la Storia della musica (Bologne 1757-1781, 3 tomes) qui nourrissent sa réputation d'érudit ${ }^{34}$, le compositeur et théoricien bolonais échange de nombreuses lettres avec des musiciens actifs dans la péninsule italienne mais aussi à l'étranger. En outre, en tant que responsable de l'Accademia Filarmonica de Bologne, il est souvent sollicité par les musiciens.

Si Sabbatini cherche le soutien de Martini, il met également en œuvre des processus de légitimation dans le milieu musical romain.

La première réaction de Sabbatini face au jugement négatif de Casali est de dresser une note informative (informazione sopra alle opposizioni fatte dal Sig. Casali) au cardinal protecteur de la confrérie, Giovanni Francesco Albani, et à son neveu, Monseigneur Giuseppe Andrea Albani (1750-1834) qui occupe la charge de Préfet de la Congrégation ${ }^{35}$.

(33) Sur la figure de Giovanni Battista Martini, voir notamment Leonida Busi, Il padre G.B. Martini musicisita-letterato del secolo XVIII, Bologne, Zanichelli, 1891 ; Angelo PoMPILIO, Padre Martini : musica e cultura nel Settecento Europeo, Florence, Olschki, 1987 ; Piero Mioli, Padre Martini. Musicista e musicografo da Bologna all'Europa (1706-1784), Lucques, Libreria Musicale Italiana, 2006.

(34) Voir la notice "MARTINI, Giovanni Battista", Dizionario Biografico degli Italiani on line - Volume 71 (2008), notice d'Elisabetta Pasquini.

(35) Lettre de Luigi Antonio Sabbatini à Giovanni Battista Martini, Rome, 9 juin 1784. 
Le compositeur espère ainsi recevoir le soutien des protecteurs de la congrégation, détenant un pouvoir institutionnel, pour contrer le jugement de Casali ${ }^{36}$, tout en ayant conscience qu'il doit compléter son initiative en ayant recours à l'expertise d'autres musiciens. Ainsi, dans sa lettre du 9 juin 1784, il explique avoir sollicité l'avis de plusieurs maîtres de chapelle exerçant à Rome pour évaluer les compositions de ses élèves ${ }^{37}$. Dans la note informative rédigée par Sabbatini, on apprend que sept compositeurs ont d'abord reconnu la validité des compositions des deux jeunes candidats, par un « jugement dépassionné » et de façon « unanime $»^{38}$.

Pourtant, si les maîtres de chapelle sollicités se sont montrés verbalement favorables aux deux élèves de Sabbatini, ils se rétractent finalement devant l'autorité de Casali ${ }^{39}$. Les maîtres de chapelle se sont ainsi laissés convaincre par les arguments de Casali, ce qui complique la tâche de Sabbatini qui ne dispose plus de soutien dans le milieu musical local. L'aide de Giovanni Battista Martini lui est donc d'autant plus nécessaire pour remporter la cause, raison pour laquelle il se montre particulièrement attentif aux propositions de ce dernier.

\section{Giovanni Battista Martini : défendre la dignité de la profession}

Dans la lettre du 16 juin 1784 où il s'engage à défendre les deux candidats et Sabbatini, Martini précise qu'il a écrit à Casali sur un ton amical tout en le pressant d'abandonner cette affaire. Il se montre aussi très précis sur la marche à suivre si ce dernier s'obstine. Dans le cas d'un refus de la part de Casali, Martini est disposé à utiliser la presse écrite pour faire savoir à tous que Casali a commis une erreur de jugement :

« J'ai résolu d'écrire à Monsieur Casali de façon amicale, l'avertissant de se retirer de son étrange engagement [...] La lettre que je lui ai écrite parle clairement, mais en ami, parce qu'il a été l'un de mes élèves [...] Nous verrons bien quel effet aura cette lettre, mais si celui-ci s'obstine à

(36) Sur les systèmes de clientélisme et de patronage dans le domaine musical, voir notamment David HenNebelle, « Nobles, musique et musiciens à Paris à la fin de l'Ancien Régime : les transformations d'un patronage séculaire (1760-1780) », Revue de musicologie, 2001, vol. 87, n 2 , p. 395-418 ; Roger FREITAS, Portrait of a Castrato : politics, patronage, and music in the life of Atto Melani, Cambridge, New York, Melbourne, Cambridge University Press, 2009.

(37) Lettre de Luigi Antonio Sabbatini à Giovanni Battista Martini, Rome, 9 juin 1784.

(38) Cette note informative a été transcrite dans la lettre de Luigi Antonio Sabbatini à Giovanni Battista Martini, Rome, 14 juillet 1784.

(39) Lettre de Luigi Antonio Sabbatini à Giovanni Battista Martini, Rome, 26 juin 1784 : « les maîtres de chapelle de Rome auxquels j'avais fait juger les compositions n'eurent pas le courage de les approuver par écrit ». 
ne pas vouloir céder, je suis prêt à défendre les candidats, en démontrant que l'examinateur est incapable de juger, et si je dois le faire, je veux que ce soit publié dans la presse $»^{40}$.

On retrouve ici sous la plume de Martini l'importance de la communication et de la collaboration dans le développement du savoir qui caractérise l'idéal de la République des Lettres ${ }^{41}$. Présentant le visage d'un savant $«$ communicatif ${ }^{42}$, Martini veut rendre publique l'affaire et utiliser cette publicité comme moyen de pression pour convaincre Casali d'abandonner la cause.

Dans la première lettre qu'il envoie à Casali à la fin du mois de juin, le musicien met également en place un stratagème habile dans le but de tester les capacités musicales du destinataire. En effet, Martini demande à Casali de se prononcer sur une composition musicale que l'on vient de lui soumettre, sans indiquer le nom de son auteur.

Le 13 juillet 1784, Padre Martini reçoit la réponse de Giovanni Battista Casali rédigée quelques jours plus tôt. Ce dernier commence sa lettre en rendant son jugement sur la composition que Martini lui a demandé d'examiner et se montre très critique : «En définitive, selon moi, elle [la composition] me semble de mauvaise harmonie, et de mauvais effet notamment avec les nombreux tritons que l'on trouve ${ }^{43}$.

Pourtant, la composition anonyme envoyée n'est autre qu'une composition de Giovanni Pierluigi da Palestrina, l'un des principaux représentants de l'école romaine. Par sa réponse, Casali révèle qu'il n'a pas reconnu le prestigieux compositeur, ce qui le discrédite fortement. Dans la lettre qu'il envoie à Sabbatini pour l'informer de cette réponse, Martini condamne l'audace insensée de Casali qui a osé «critiquer et corriger Palestrina » et se montre plus que jamais déterminé à le désavouer, espérant «qu'avec le temps et la patience », ils vaincront ${ }^{44}$.

Dans la réponse qu'il envoie à Casali le 14 juillet 1784, Martini n'exprime aucune forme d'animosité envers lui. En revanche, dans les

(40) Lettre de Giovanni Battista Martini à Luigi Antonio Sabbatini, Bologne, 16 juin 1784.

(41) À ce sujet, voir Françoise WAQUET, « Qu'est-ce que la République des Lettres ? », op. cit., p. 492-493.

(42) « Fondé sur la collaboration, le savoir moderne implique la communication [...] il n'est pas pour un savant de plus belle qualité que d'être "communicatif" ». Ibidem, p. 492.

(43) Lettre de Giovanni Battista Casali à Giovanni Battista Martini, Rome, 7 juillet 1784. En musique, un « triton » est l'autre nom donné à l'intervalle de quarte augmentée, ou encore de quinte diminuée. Ce nom est dû au fait que cet intervalle fait exactement trois tons (ce qui donne triton), soit une demi-octave. (minute).

(44) Lettre de Giovanni Battista Martini à Luigi Antonio Sabbatini, Bologne, 17 juillet 1784 
lettres qu'il envoie à Sabbatini, il se montre très hostile. Le 28 juillet 1784, par exemple, il dénonce clairement l'incompétence de Casali, manifeste dans le fait que ce dernier n'ait pas été capable de reconnaître l'écriture de Palestrina :

« Palestrina, non seulement à Rome, mais dans toute l'Europe a toujours été considéré comme étant le meilleur Maître de tous les compositeurs, et que je sache il n'a jamais été critiqué par personne. Monsieur Casali, qui exerce en juge suprême à Rome, n'est peut-être pas obligé de reconnaître l'écriture et le style de Palestrina ? C'en est trop ! $»^{45}$.

Giovanni Pierluigi da Palestrina se présente en effet comme une référence pour la musique religieuse, le fondement d'une tradition, d'une représentation partagée de l'histoire de la musique ${ }^{46}$. La critique de Casali remet en cause cette image et, à travers elle, une certaine conception de la profession, ce qui renforce clairement l'hostilité de Martini à son égard.

Avant même de recevoir la première réponse de Casali, en date du 7 juillet, Martini expose àSabbatini une stratégie si Casali refuse. Le 3 juillet 1784, Martini se montre déterminé à poursuivre sa défense et précise qu'il sera peut-être nécessaire d'avoir recours à la justice :

« Vu que Monsieur Casali insiste pour ne pas vouloir les admettre, voici le remède. Faites rédiger une appellation formelle de la main d'un notaire par les deux candidats, avec laquelle vous appelez en justice Monsieur Casali, et qu'ils se remettent tous deux à mon jugement, dans ce cas je ferai connaître à Monsieur Casali qu'il n'est pas capable de juger, lui manquant toutes les qualités qu'il incombe à un juge. Et si j'ai besoin d'émettre ce jugement, je veux qu'il soit publié, afin que le public soit informé du tort manifeste, et de son incapacité à juger $[\ldots] \gg^{47}$.

Martini annonce ainsi à Sabbatini qu'il dispose d'arguments conséquents contre Casali. Il demande néanmoins à Sabbatini de se renseigner pour savoir si les candidats ont reçu des instructions sur les règles d'écriture pour la réponse de la fugue.

(45) Lettre de Giovanni Battista Martini à Luigi Antonio Sabbatini, Bologne, 28 juillet 1784.

(46) Sur le mythe de Palestrina qui naît au XVII ${ }^{\mathrm{e}}$ siècle, voir Lorenzo BIANCONI Il Seicento, Torino, EDT, 1982, p. 115-117. (minute).

(47) Lettre de Giovanni Battista Martini à Luigi Antonio Sabbatini, Bologne, 3 juillet 1784 
Dans une lettre datée du 10 juillet $1784^{48}$, Sabbatini précise que les statuts de la congrégation ne mentionnent pas les règles de composition et qu'aucune directive n'a été donnée aux candidats avant de composer. Tous les maîtres de chapelle qu'il a rencontrés lui assurent qu'aucun document ne précise cette règle mais aucun d'eux n'accepte de s'engager par écrit. Sabbatini ajoute que les archives de l'institution conservent des compositions d'examen de candidats reçus bien que les réponses de la fugue ne soient pas écrites dans la bonne tonalité. Ces documents révèlent la partialité de l'examen et peuvent devenir une preuve valide pour un éventuel recours en justice.

Parallèlement, le maître de chapelle bolonais continue d'exprimer son soutien à Sabbatini et condamne l'entêtement de Casali. Pour contrer Casali, Padre Martini propose trois solutions à Sabbatini. Ce dernier devait choisir celle qu'il jugerait la plus pertinente :

«I. Je le citerais à comparaître, en déclarant son jugement nul, parce qu'il n'a pas en lui les qualités qu' un juge requière, en plus du fait qu'il est frauduleux, et de mauvaise foi. II. Je donnerais un Mémorial au Pape, en y exposant le tort que Monsieur Casali a fait à ces deux jeunes gens, en ne voulant pas les approuver sans raison, et seulement de mauvaise grâce, et si jamais vous estimez opportun que je fasse une attestation en faveur des deux candidats, laquelle soit mise sous les yeux du Pape, je suis prêt. III. Je citerais Casali, l'obligeant à donner la raison pour laquelle il ne veut pas approuver les deux candidats $»^{49}$.

Les trois propositions de Martini portent toutes préjudice à Casali. Martini se montre résolu à poursuivre Casali car l'attitude et les discours de ce dernier s'opposent à la conception de la profession musicale et du rôle des examinateurs qu'il soutient. L'argumentaire de Martini est construit autour d'une défense de l'art musical et des règles traditionnelles qui s'y rapportent. Son avis se fonde sur une connaissance de l'histoire de la profession, et des contributions de ses prédécesseurs parmi les plus réputés, dont celle de Palestrina. Cette revendication d'une « tradition professionnelle » est un élément qui doit être porté à l'actif de la construction d'une certaine idée de la profession ${ }^{50}$. Attaché à la dimension collective

(48) Lettre de Luigi Antonio Sabbatini à Giovanni Battista Martini, Rome, 10 juillet 1784.

(49) Lettre de Giovanni Battista Martini à Luigi Antonio Sabbatini, Bologne, 10 juillet 1784.

(50) Sur la place de la théorie et de l'historiographie de l'art dans les processus de professionnalisation, cf. Bram KEMPERS, Peintres et mécènes de la Renaissance italienne, Paris, Gérard Monfort, 1997, traduction française de l'édition originale de 1987. 
du jugement, Martini rejette également l'attitude de Casali, autoritaire et poursuivant un intérêt personnel, au nom d'un savoir qui doit être soumis au jugement des différents membres de la communauté musicale et à un échange ouvert des opinions.

\section{Giovanni Battista Casali : une vision corporative et hiérarchique}

Qu'en est-il alors de la défense construite par Casali ? Le compositeur romain, quant à lui, utilise dans un premier temps des ressources locales pour se défendre des attaques et contrer les initiatives de Sabbatini. Le 3 juillet 1784, celui-ci indique que Casali fait circuler un document adressé aux maîtres de chapelle, dont il ne connaît pas le contenu ${ }^{51}$. On apprend en effet plus tard que Casali a réussi à rallier les maîtres de chapelle à sa cause :

« J'imagine qu'il [Casali] lui aura envoyé le papier avec les approbations des maîtres, lequel indique que tous conviennent que la réponse n'est pas tonale, ce qui ne change rien, et les mêmes maîtres qu'il a cherché à faire souscrire, sont ceux auxquels j'ai moi-même fait voir les deux fugues auparavant, et tous les jugèrent bonnes, mais maintenant, avec les paroles de Casali ils ont tous baissé la tête dans l'espoir de pouvoir obtenir des remplacements dans ses églises, ou bien parce qu'ils ne savaient pas répondre aux arguments de Casali $»^{52}$.

Sabbatini, déçu du revirement des maîtres de chapelle, décrit la position hégémonique qu'occupe Casali et l'influence sans borne qu'il exerce. Selon lui, les maîtres de chapelle se rallient à son jugement dans l'espoir de le remplacer dans les chapelles où ce dernier dispose d'un poste de titulaire, ce qui confirme la vive compétition qui se joue dans le milieu romain pour accéder à ces emplois.

Des écrits de Casali au sujet de cette affaire, seule la lettre de réponse à Padre Martini datée du 7 juillet 1784 nous est parvenue ${ }^{53}$.

Le compositeur mentionne les compositions d'examen des deux candidats et les problèmes qu'elles présentent.Casali signale aussi que l'affaire a créé une grande confusion à Rome. Il mentionne la témérité d'un

(51) Lettre de Luigi Antonio Sabbatini à Giovanni Battista Martini, Rome, 3 juillet 1784 : « je ne sais pas ce que contient le feuillet, mais je sais par ailleurs, qu'à l'endroit où étaient présents un religieux et deux cavaliers il a dit que, lui vivant, ces deux [candidats] ne passeraient pas ».

(52) Lettre de Luigi Antonio Sabbatini à Giovanni Battista Martini, Rome, 10 juillet 1784.

(53) Padre Martini recopia la lettre de Casali qu'il avait reçue le 13 juillet dans la lettre qu'il adressa à Sabbatini, Bologne, 14 juillet 1784. 
des élèves de Sabbatini et signale que des insultes et des paris circulent et compromettent la réputation des maîtres de chapelle ${ }^{54}$.

Son plaidoyer suit une logique de garantie du monopole, basée sur la défense de la congrégation de Sainte Cécile, sur ce qui la distingue, et sur la mise en valeur de sa position dominante pour exercer un contrôle de la profession. Il expose une défense du corporatisme en construisant son discours autour du respect des règles de composition relatives à la fugue qui, selon lui, figurent dans les statuts :

« L'obligation de répondre dans la même tonalité est tellement évidente, qu'à celui qui ne respecterait pas cela et écrirait comme bon lui semble, je dirai que cela fait partie des statuts de notre Congrégation observés depuis plus de cinquante ans, statuts confirmés par le bref pontifical de Clément $\mathrm{XI}[\ldots] »^{55}$.

Pour contrer l'argumentation de Padre Martini qui a comparé ce conflit aux difficultés rencontrées par Casali à Bologne à l'occasion de son admission à l'Accademia Filarmonica, le compositeur explique que son refus ne témoigne pas d'une attitude conservatrice ou protectrice :

«Ici, on n'exerce pas par passion, ou par crainte de Dieu témoin, comme cela fut le cas pour ma personne à Bologne. Parce qu'en plus des trois églises pour lesquelles je reçois un salaire mensuel, je sers aussi cinquante autres églises dont les principales, et par la grâce de Dieu en ville j'ai fait en sorte que je n'ai pas l'ombre d'une peur que ces personnes m'enlèvent les fonctions musicales $»^{56}$.

Le compositeur, précisant qu'il cumule de nombreux postes dans les chapelles musicales, se défend d'agir selon des intérêts contraires à ceux de la profession.

Dans la fin de la lettre, Casali tente enfin, sur un ton presque menaçant, de dissuader Padre Martini de toute intervention :

«Par ce conseil en tant que bon Ami, je vous éviterais la fatigue des documents à préparer pour cette défense, car vous serez perdant, parce que vous aurez une réponse de six ou sept lignes ; si vous la publiez, nous aussi ; si vous la diffusez en Europe, moi je corresponds avec La Havane en Amérique, à Lima au Pérou, à Séville, dans les Flandres, en Allemagne,

(54) Lettre de Giovanni Battista Casali à Giovanni Battista Martini, Rome, 7 juillet 1784.

(55) Ibidem.

(56) Ibid. 
à Turin, à Venise [...] vous montrerez votre savoir, mais vous ne servirez pas l'intérêt des candidats parce qu'un décret d'Assemblée générale sera émis pour que ceux-ci ne puissent plus jamais se présenter à l'examen ${ }^{57}$.

Casali avertit Martini qu'il est également prêt à se défendre dans le cas d'une attaque par la presse. Non seulement il s'appuiera sur l'influence de la congrégation romaine, mais il affirme également disposer d'un réseau international qui s'étend jusqu'en Amérique espagnole, lui permettant de faire valoir son autorité qu'il juge supérieure à celle de son ancien enseignant, limitée, selon lui, à l'échelle européenne. Le musicien se dit donc prêt à solliciter des soutiens externes. À la fin de la lettre, il propose néanmoins aux deux candidats de repasser l'examen pour mettre fin à cette affaire $^{58}$. L'attachement du musicien à la pratique de l'examen s'exprime une fois encore ; elle lui permet de contrôler les accès aux postes de maîtres de chapelle et d'exercer ainsi une hégémonie sur le marché local.

\section{Une issue incertaine}

Dans sa réponse datée du 14 juillet 1784, Padre Martini, «persuadé de la dignité, et du respect que doivent avoir les Professeurs de musique entre eux quel que soit leur rang ${ }^{59}$, conseille à Casali, sur un ton amical et respectueux, de mettre fin à la controverse à l'amiable, notamment en cherchant un médiateur compétent capable de régler le conflit.

Pourtant, si le ton de Martini est relativement apaisé dans sa réponse à Casali, il se montre intransigeant envers lui dans les lettres qu'il adresse à Sabbatini. Il demande à ce dernier de lui fournir tous les documents qui peuvent l'aider à préparer sa défense et dont Sabbatini lui a parlé dans les lettres précédentes, dont les compositions des précédents candidats admis, afin que Casali « ouvre progressivement les yeux » sur cette affaire ${ }^{60}$.

Martini se renseigne également auprès de Sabbatini pour savoir s'il dispose de soutiens dans le milieu musical romain. Il s'inquiète notamment de l'avis du compositeur et théoricien de la musique Antonio Eximeno ${ }^{61}$. On peut imaginer qu'il recherche un soutien local puissant, notamment

(57) Ibid

(58) Ibid.

(59) Lettre de Giovanni Battista Martini à Giovanni Battista Casali, Bologne, 14 juillet 1784.

(60) Lettre de Giovanni Battista Martini à Giovanni Battista Sabbatini, 17 juillet 1784.

(61) Antonio Eximeno Pujades (1729-1809), originaire d'Espagne, exerce à Rome depuis la fin des années 1760. Il devient en 1773 membre de l'Académie littéraire de l'Arcadie en 1773. Il est notamment l'auteur de l'ouvrage Dell'origine e delle regole della musica, con la storia del suo progresso, decadenza e rinnovazione (Rome, 1774). 
parmi les musiciens qui jouissent d'un prestige certain dans le monde des lettres.

Dans sa lettre datée du 21 juillet 1784, Martini, dans l'attente de la réponse de Casali à la lettre qui propose un tiers pour régler le conflit, pense que, si Casali et les maîtres de chapelle romains le refusent, la solution d'avoir recours au Cardinal protecteur de la Congrégation serait sans doute la « solution la plus juste $»^{62}$ et la plus judicieuse pour mettre fin au conflit, tout en ayant conscience que cela nuirait gravement à la réputation de Casali.

Dans la lettre du 21 juillet 1784, Sabbatini précise qu'il est prêt à poursuivre Casali en justice et que le cardinal Albani est disposé à le soutenir :

«Moi, pendant ce temps, ayant pensé qu'il ne voudrait pas envoyer les diplômes, ce qui m'a en effet été confirmé ce matin, puisqu'il s'est exprimé pour dire qu'il ne voulait pas les donner, je m'étais déjà assuré auprès du Cardinal Albani de la permission de le faire comparaître en lui exposant les raisons [...] Si bien que maintenant, j'attends sa confirmation, et juste après je le fais comparaître, alors que sur les trois points que vous me signifiez, celui-ci me paraît le plus efficace $[\ldots] »^{63}$.

Il attend donc le jugement de Martini pour appuyer son argumentation et exprime sa détermination pour remporter l'affaire.

Sabbatini, dans la lettre du 24 juillet 1784, reprend point par point les arguments avancés par Casali pour les contester. La défense de Casali qui se base sur les statuts de la Congrégation est « démontée » par les examens de composition de candidats reçus que Sabbatini a en sa possession, celle de Rosa Maria Coccia, de Benedetto Alimenti et d'Antonio Aurisicchio. Selon lui, d'autres compositions conservées dans les archives sont tout aussi inadaptées. Les copies des compositions d'examen de certains candidats admis bien qu'elles ne fussent pas tonales étaient une preuve de la partialité de Casali. Sabbatini s'insurge aussi devant le manque d'informations données par les examinateurs et précise que l'argument avancé par Casali au sujet de la présence des règles de la fugue dans les statuts n'a pas de sens :

«Si ce point est présent dans les statuts pourquoi ne le font-ils pas savoir à tous ceux qui passent l'examen, d'autant plus qu'ils sont très

(62) Lettre de Giovanni Battista Martini à Luigi Antonio Sabbatini, Bologne, 21 juillet 1784.

(63) Lettre de Luigi Antonio Sabbatini à Giovanni Battista Martini, Rome, 21 juillet 1784. 
nombreux, ceux-ci n'ayant pas été avertis ; de même que les quatre autres qui allèrent à l'examen avant eux [...] Où est donc cette règle qui doit être observée avec exactitude ? Vous voyez déjà que, de toutes les façons, il [Casali] se met à terre avec des arguments de la sorte ${ }^{64}$.

Sabbatini n'a aucune confiance en Casali et se montre déterminé à le poursuivre en justice, comme prévu.

Dans les deux dernières lettres qu'il rédige avant sa mort, datées du 28 et du 31 juillet 1784, Martini exprime aussi son désir de voir juger Casali, en rappelant qu'il a déshonoré la profession non seulement par sa conduite, mais en osant critiquer aussi Palestrina :

« Je suis et serai constant dans l'opinion, que l'on doive citer Monsieur Casali, dénonçant son mauvais jugement, et de demander à l'Excellentissime Monsieur le Cardinal Albani de trouver un tiers [...] Je reste étonné, et surpris de voir les compositions approuvées par le même Casali : Pauvre Rome, dans quel état déplorable a-t-elle été réduite ! je ne pensais pas à un excès de cette envergure. Je joins ici une attestation qui approuve les susdites compositions de vos élèves, afin que, si vous le jugez utile, vous puissiez la présenter aux yeux de Votre Excellence afin qu'il voit la faiblesse de Monsieur Casali. Passion, malignité et ignorance sont les bases sur lesquelles s'est fondée sa conduite $»^{65}$.

Si Sabbatini et Martini se montrent prêts à poursuivre Casali dans cette affaire, la mort du maître de chapelle bolonais empêche inévitablement une prompte résolution. Sans le dossier préparé par Martini, la défense de Sabbatini est nettement plus fragile. Le musicien essaie, dans les mois qui suivent, d'obtenir l'appui de l'Accademia Filarmonica di Bologna en correspondant avec Stanislao Mattei, l'ami de Martini.

Ce conflit, reconstruit à partir des témoignages des trois principaux protagonistes de l'histoire, révèle les principaux enjeux qui se déclinent autour de la pratique de l'examen. Ce moyen de sélection semble approuvé de tous. Il apparaît bien comme un signe de professionnalisation, en permettant aux maîtres de chapelle de juger collégialement, en interne et selon des critères techniques, du niveau de pratique des postulants. Il renforce l'identité collective des musiciens, par le partage de critères de compétence. En revanche, le différend porte sur la façon dont il doit

(64) Lettre de Luigi Antonio Sabbatini à Giovanni Battista Martini, Rome, 24 juillet 1784.

(65) Lettre de Giovanni Battista Martini à Luigi Antonio Sabbatini, Bologne, 31 juillet 1784. 
être conduit pour en garantir les effets bénéfiques sur la profession. Les conditions et la pratique de l'examen ne sont jamais neutres ; loin de n'avoir d'implications que pour l'entrée dans une carrière, elles mettent en jeu les positions les mieux établies au sein de la profession. Le monopole de Casali est fortement contesté par Padre Martini et Sabbatini. Pour ces derniers, la mise en œuvre collégiale du déroulement de l'examen et de la prise de décision doit être strictement respectée pour éviter toute captation personnalisée de la légitimation professionnelle. De son côté, Casali cherche à renforcer, sans doute à son profit, la congrégation comme pôle exclusif de reconnaissance.

En outre, les compositeurs, en affirmant publiquement leur capacité à juger de la validité ou non de l'examen, manifestent leur autorité dans l'univers professionnel auquel ils appartiennent. On observe que Casali se vante de pouvoir faire jouer ses protections à l'échelle internationale, au-delà même de l'Europe, alors que les fondements de son jugement sont contestés localement. Sabbatini indique aussi que la résolution du conflit en sa faveur est essentielle à sa réputation. Les candidats écartés sont ses élèves, et son crédit en tant que maître de musique se trouve donc fortement engagé dans cette affaire. Le jugement autour de l'examen leur donne ainsi l'occasion de renforcer leurs protagonistes, et de construire leur sphère d'influence, dans un milieu musical compétitif et en dehors de ce monde, en se signalant comme de puissantes références pour qui veut entreprendre ce type de carrière.

Élodie ORIOL

UMR 7303 Telemme, Aix-Marseille Université/CNRS MMSH - 5, rue du Château de l'Horloge BP 647 - 13094 Aix-en-Provence Cedex 2 elodieoriol@hotmail.com 